\title{
Optimization of Silicon and Quartz MEMS Microheater for Chemoresistive Gas Sensors
}

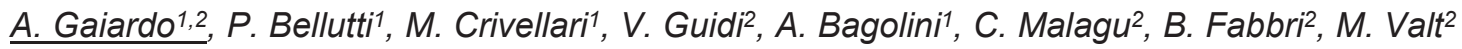 \\ ${ }_{1}^{1}$ MNF- Micro Nano Facility, Bruno Kessler Foundation, Via Sommarive 18, 38123 Trento, Italy \\ ${ }^{2}$ Department of Physics and Earth Science, University of Ferrara, Via Saragat 1/c, 44122 Ferrara, \\ Italy \\ Corresponding author's e-mail address: gaiardo@fbk.eu
}

\begin{abstract}
:
In the last years, the research in the gas sensor field experienced a significant boost. Gas sensors represent the crucial elements in gas monitoring systems and olfactory systems for several applications: environmental monitoring, safety and security, quality control of food production, medical diagnosis and so on. From the point of view of the gas sensing design, the substrate plays a fundamental role, because acts as a heater, mechanical support and transducer of the sensor response. The application of MEMS technology for the fabrication of silicon device with low power consumption has offered new opportunities for innovative gas sensor design. In this work, we studied different approaches to realize an adapt silicon and quartz microheaters for chemoresistive gas sensors, available for high operating temperatures $\left(650^{\circ} \mathrm{C}\right)$ through the MEMS technology. Our study is focused on a fabless approach to microheater fabrication, because of the lack of a cheap CMOS compatible process.
\end{abstract}

Key words: Chemoresistive gas sensors, low-power consumption, MEMS device, microheater, fabless approach.

\section{Introduction}

Microheater, a device identified as microelectro-mechanical system - MEMS, became a key component in chemical sensors. It acts as a micro hotplate that, combined with sensitive sensing element, provides the sensor [1]. The benefits of using silicon and quartz microheater platforms include: reduced power consumption compared to traditional thick-film gas sensors on other substrates, reduced size and excellent temperature uniformity. Furthermore, silicon based microheater can allow compatibility with CMOS process, thus enabling possible integration of the sensor with the signal processing electronic on the same die [2]. Although alumina is still today the most widely used substrate, especially in research, silicon and quartz are the best promising alternatives [3].

\section{Experimental}

In order to assess a reliable microdevice for this application field, in this work, we studied the different processing steps required to obtain suitable silicon and quartz microheaters (Fig. 1). The operative characteristics of these devices strongly depend on many realization parameters, such as: layout of the devices, types of metals used as a heater and interdigitated contacts, type of insulator and heat treatment to be adopted during the microfabrication.

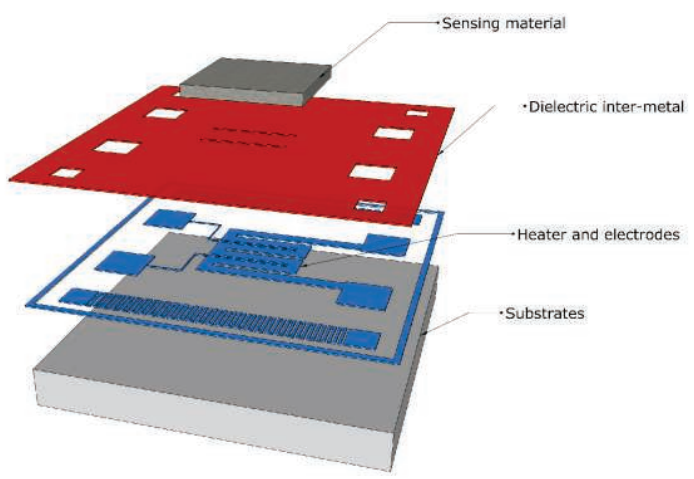

Fig. 1. Design of the microfabrication process.

This work is focused on the design topics. For these reasons, various types of layouts were designed and then realized to verify their impact on the sensor performances (Fig. 2). Regarding silicon devices, and initial passivation layer of $\mathrm{SiO}_{2} / \mathrm{Si}_{3} \mathrm{~N}_{4} / \mathrm{SiO}_{2}$ was deposited onto silicon wafers, by means of thermal growth and 
LPCVD techniques. The metal used to realize the contact was a double layer of platinum (120 $\mathrm{nm})$ and titanium $(10 \mathrm{~nm})$, used as adhesion layer, for both silicon and quartz processes. The metal layers were deposited by a physical vapor deposition system. $\mathrm{SiO}_{2}$ and $\mathrm{Si}_{3} \mathrm{~N}_{4}$ have been used to electrically isolate the microheater from the interdigitated contacts and the CMOS sensitive film.

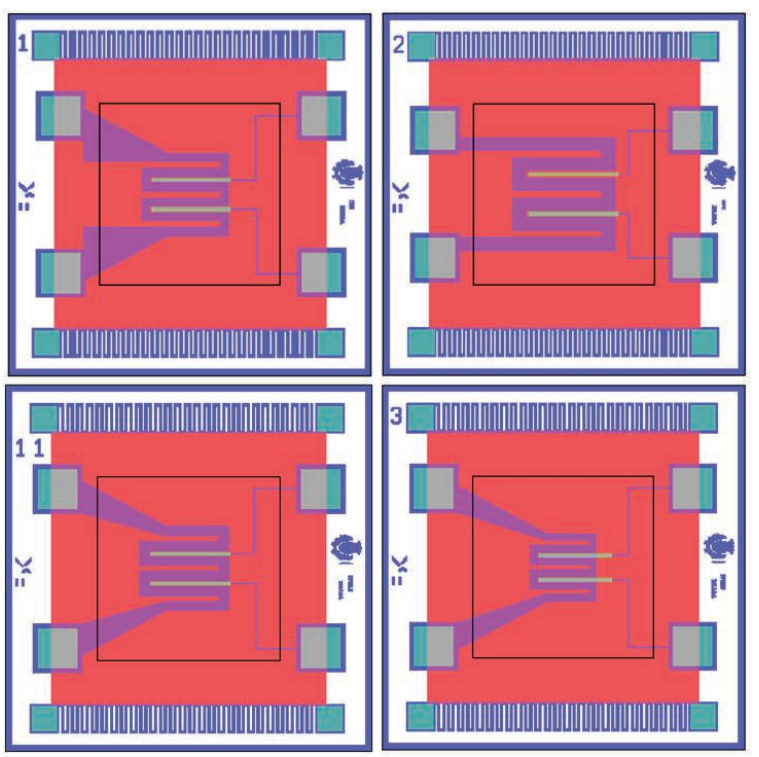

Fig. 2. Different layouts realized and investigated.

Dielectric layers were deposited by PECVD technique by using different deposition parameters. In the following, thermal treatments for system stabilization were introduced and different temperatures has been adopted to verify their impact on device stability (Fig. 3).

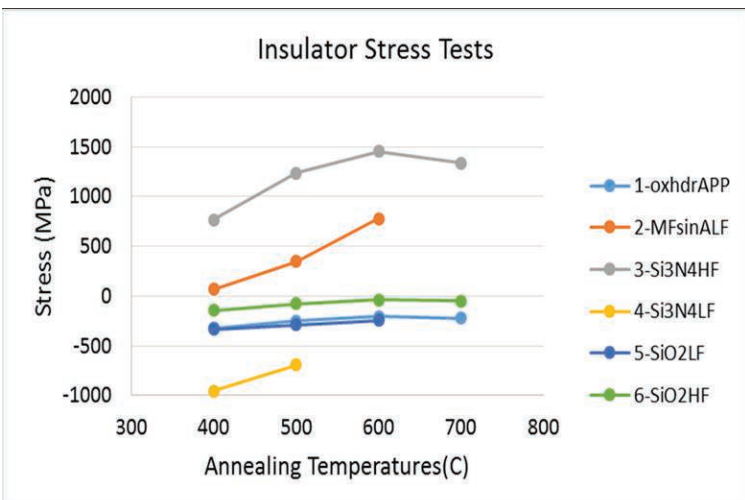

Fig. 3. Different layouts realized and investigated.

Specific stress tests were performed to characterize the stress content of the dielectric film vs annealing temperature. Afterwards, the silicon devices were dipped in a TMAH solution for etching the silicon to obtain a thin dielectric membrane, supporting the heating elements, required condition to ensure the low power consumption. Eventually, the devices obtained were connected to a T039 package through ball bonding process, verifying the adhesion strength with pull and scratch tests.

Then, $\mathrm{SnO}_{2}$ sensing material was deposited on both silicon and quartz microheaters, by means of screen printing technique (Fig. 4). were characterized to analyse the power consumption used to thermo-activated the gas sensing material (Fig. 4).

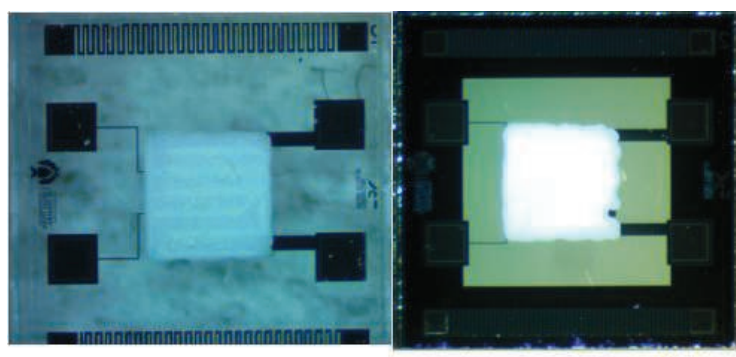

Fig. 4. $\mathrm{SnO}_{2}$ sensing material deposited onto quartz (left) and silicon (right) microheaters.

The gas sensing characterization of $\mathrm{SnO}_{2}$ sensing material vs. $20 \mathrm{ppm}$ of $\mathrm{CO}$, thermoactivated at $450^{\circ} \mathrm{C}$, highlighted the repeatability of the sensing responses, combined with a high thermal and mechanical stability of both microheaters (Fig. 6).

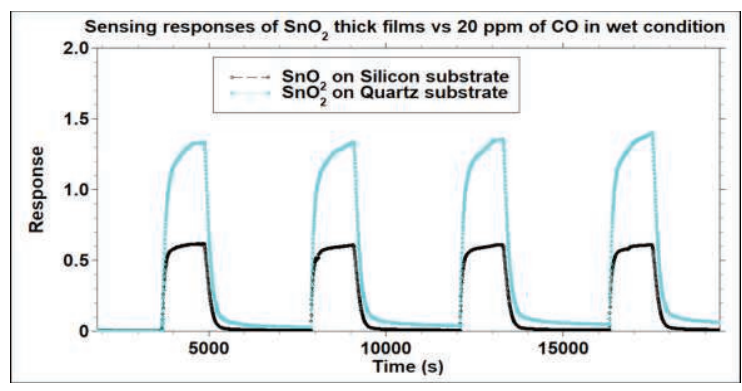

The power consumption of both microheaters was lower than common commercial alumina substrates, with a value of tens $\mathrm{mW}$ for silicon substrate.

In this study, we developed a possible fabless process to produce cheap and low power consumption silicon and quartz microheaters.

\section{References}

[1] M. A. Andio, P.N. Browning, P.A. Morris, S.A. Akbar, Comparison of gas sensor performance of $\mathrm{SnO}_{2}$ nano-structures on microhotplate platforms. Sensors and Actuators B: Chemical, 165, 13-18 (2012); doi: 10.1016/j.snb.2011.12.045.

[2] S. Maity, A. Kumar, Fabrication of microheater and inter-digitedelectrodes on silicon platform for low power integrated gas sensor applications. $J$ Res Electr Electron Eng, 2(4), 6-10 (2013); doi: $10.1007 / \mathrm{s} 00542-015-2477-8$

[3] J.W. Gardner, P.K. Guha, F. Udrea, J.A. Covington, CMOS interfacing for integrated gas sensors: A review IEEE Sensors Journal, 10 (12), 1833-1848 (2010); doi:

10.1109/JSEN.2010.2046409 